\title{
Emodin inhibits extracellular matrix synthesis by suppressing p38 and ERK1/2 pathways in TGF- $\beta 1$-stimulated NRK-49F cells
}

\author{
BIN ZHU, YI LIN, CAI-FENG ZHU, XIAO-LING ZHU, CHEN-ZHAO HUANG, \\ YING LU, XIAO-XIA CHENG and YONG-JUN WANG
}

\begin{abstract}
Department of Nephrology, Hangzhou Hospital of Traditional Chinese Medicine (Hangzhou Guangxing Hospital), Zhejiang Chinese Medical University, Hangzhou, Zhejiang, P.R. China
\end{abstract}

Received November 2, 2010; Accepted February 4, 2011

DOI: $10.3892 / \mathrm{mmr} .2011 .444$

\begin{abstract}
Emodin has been demonstrated to inhibit the fibrotic process in chronic renal disease, but its mechanisms have yet to be fully elucidated. This study was carried out to investigate the effects of emodin on extracellular matrix (ECM) synthesis in TGF- $\beta 1$-stimulated NRK-49F cells. NRK-49F cells stimulated with TGF- $\beta 1$ were incubated with various concentrations of emodin. ECM proteins, including collagen type III and fibronectin, were detected using ELISA. ERK1/2, p38 and JNK phosphorylation were measured by Western blotting. p38, ERK1/2 and JNK were respectively inhibited with the specific inhibitors SB203580, PD98059 and SP600125. Emodin slightly inhibited the expression of fibronectin and collagen type III in NRK-49F cells without TGF- $\beta 1$ treatment, and significantly suppressed fibronectin and collagen type III secretion in TGF- $\beta 1$-stimulated NRK-49F cells. ERK1/2 and p38 specific inhibitors, but not JNK inhibitor, suppressed the TGF- $\beta 1$-induced expression of fibronectin and collagen type III. Our previous study demonstrated that there was no crosstalk between ERK1/2, p38 and JNK signals in TGF- $\beta 1$-stimulated NRK-49F cells. Here, we found that emodin inhibited the phosphorylation of ERK1/2 and p38 significantly, but did not suppress the phosphorylation of JNK. In summary, emodin suppresses fibronectin and collagen type III expression via the inhibition of ERK1/2 and p38 phosphorylation in TGF- $\beta 1$-stimulated NRK-49F cells.
\end{abstract}

\section{Introduction}

Emodin, 3-methyl-1,6,8-carboxyl-anthraquinone, is an anthraquinone derivative isolated mainly from the Chinese herb Rheum palmatum. It has been demonstrated to inhibit

Correspondence to: Dr Bin Zhu, Department of Nephrology, Hangzhou Hospital of Traditional Chinese Medicine (Hangzhou Guangxing Hospital), Zhejiang Chinese Medical University, Hangzhou, Zhejiang 310007, P.R. China

E-mail: zhubing@hotmail.com

Key words: emodin, mitogen-activated protein kinase, renal fibrosis, extracellular matrix the fibrosis process of various organs, including the liver, pancreas, peritoneum and kidney (1-3). Emodin suppresses p38 phosphorylation to inhibit cell proliferation and fibronectin expression in mesangial cells cultured in high-glucose solution (4). It also suppresses IL-1 $\beta$-induced extracellular matrix (ECM) synthesis in mesangial cells (5). However, its mechanism is not fully elucidated.

TGF- $\beta 1$ is a cytokine that plays an essential role in renal fibrosis in various kidney diseases to promote renal failure. It induces the synthesis of ECM protein, including collagen type III and fibronectin. The most important downstream factors mediating the ECM synthesis of TGF- $\beta 1$ are mitogen-activated protein kinase (MAPK) signals, including extracellular signal-regulated kinase (ERK), p38 MAP kinase and c-jun N-terminal kinase (JNK). ERK mediates TGF- $\beta 1$ induced synthesis of plasminogen activator inhibitor type I (6), CTGF (7) and aldsterone-stimulated collagen synthesis (8). ERK is also involved in the ECM synthesis in diabetic rats (9). p38 mediates TGF- $\beta 1$-stimulated ECM synthesis in mesangial cells (10) and proximal tubular cells (11). Angiotensin II promotes ECM synthesis by activating the ERK and p38 pathways (12). ERK, p38 and JNK are also involved in the process of renal fibrosis in UUO rats (13-15). The activation of ERK and p38 induces the synthesis of TGF- $\beta 1$ to form a vicious cycle aggravating renal fibrosis (16).

Renal fibroblasts differentiate into myofibroblasts under the persistent injury involved in the overproduction and deposition of the interstitial matrix in fibrotic kidneys. They also differentiate into myofibroblasts with the up-regulation of ECM production upon TGF- $\beta 1$ stimulation. To date, there have been no reports concerning the effects of emodin on the MAPK pathways in renal interstitial fibroblasts.

In the present study, we investigated the effects of emodin on MAPK pathway to induce ECM synthesis in TGF- $\beta 1$ stimulated NRK-49F cells, a cell line of renal interstitial fibroblasts.

\section{Materials and methods}

Materials. Primary antibodies for Western blot analysis were obtained from Cell Signaling Technology (Beverly, MA, USA). A lactate dehydrogenase (LDH) assay kit was purchased from Promega (Madison, WI, USA). An ELISA 
kit for detecting collagen type III was obtained from USCN Life (Wuhan, China). An ELISA kit for detecting fibronectin was purchased from Alpco (Salem, NH, USA). The ERK1/2 inhibitor PD98059, p38 inhibitor SB203580 and JNK inhibitor SP600125 were obtained from Calbiochem (La Jolla, CA, USA).

Cell culture. NRK-49F cells were obtained from the American Type Culture Collection (Manassas, VA, USA) and cultured in Dulbecco's modified Eagle's medium (DMEM; Gibco BRL, Paisley, UK) supplemented with $100 \mathrm{IU} / \mathrm{ml}$ penicillin, $100 \mathrm{mg} /$ $\mathrm{ml}$ streptomycin and $10 \%$ fetal calf serum (Sijiqing BioTech, Hangzhou, China) at $37^{\circ} \mathrm{C}$ in $5 \% \mathrm{CO}_{2}$ and $95 \%$ air. Confluent NRK-49F cells were quiesced by incubation in DMEM for 24 h. Lastly, confluent cells were incubated with TGF- $\beta 1$ (2.5 ng/ml; R\&D Company, Minneapolis, MN, USA) or/and emodin (Sigma-Aldrich, USA) in different concentrations.

Determination of $\mathrm{LDH}$ release in NRK-49F cells. Confluent NRK-49F cells were cultured under control and experimental conditions in 96-well plates for $24 \mathrm{~h}$. Supernatants were collected, centrifuged for $10 \mathrm{~min}$ at 2,000 x g, and assessed for LDH release using a commercially available kit according to the manufacturer's instructions. The cytotoxicity of the control and experimental samples was expressed as the percentage of $\mathrm{LDH}$ release compared to total intracellular LDH content.

Enzyme-linked immunosorbent assay (ELISA). Collagen type III and fibronectin accumulation in the supernatants was quantified using ELISA following the manufacturer's instructions. In brief, after a 24-h incubation with the compounds, the samples and standards were transferred to 96-well microplates pre-coated with specific collagen type III or fibronectin antibodies and incubated for $2 \mathrm{~h}$. After the plates were washed and decanted, biotinylated rat fibronectin or collagen type III antibodies were added to each well, followed by incubation for $60 \mathrm{~min}$. Then, streptavidin-peroxidase conjugate was added to each well and the plates were incubated for $30 \mathrm{~min}$. Subsequently, chromogen substrate was added, the plates were incubated for $10 \mathrm{~min}$, then the stop solution was added to each well. The absorbance was read at $450 \mathrm{~nm}$ in an ELISA reader. The values of each sample were normalized to the protein concentrations measured using the Bradford assay (BioRad, CA, USA).

Western blotting. Cells were washed three times with PBS, added to lysis buffer (Shengneng Biotech, Shanghai, China) and vortexed thoroughly. Protein concentrations were determined by the Bradford method. Then, $5 \mathrm{X}$ loading buffer was added to the protein and the mixture was boiled for 5 min. Protein from each sample $(80 \mu \mathrm{g})$ was divided by SDS-PAGE and transferred to a polyvinylidene difluoride membrane (Millipore, Bedford, MA, USA). The membranes were blocked with $5 \%$ skimmed milk and then incubated in primary antibody overnight. The membranes were then washed with Tris-buffered saline, followed by incubation with horseradish peroxidase-conjugated second antibody for $2 \mathrm{~h}$. Lastly, the membranes were incubated in Supersignal West Dura Extended Duration Substrate (Pierce, USA) and exposed to X-ray film.

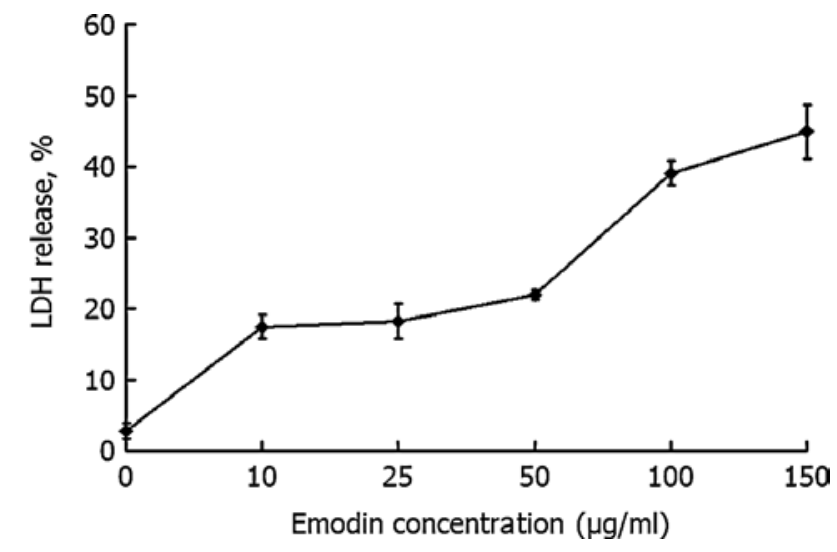

Figure 1. Effects of emodin on LDH release by NRK-49F cells. Data expressed as the means \pm SD are representative of three independent experiments with similar results. Cells were treated with various concentrations of emodin for $24 \mathrm{~h}$.

Statistical analysis. Data were expressed as the mean \pm SD and analyzed by one-way ANOVA with the LSD test for multiple comparisons. P-values $<0.05$ were considered significantly different.

\section{Results}

Cytotoxic effects of emodin by $\mathrm{LDH}$ analysis. To determine the optimal concentration of emodin to be applied in NRK-49F cells, an LDH test of various concentrations of emodin $(0-150 \mu \mathrm{g} / \mathrm{ml})$ was performed. The $\mathrm{LDH}$ release increased to $\sim 21 \%$ in $50 \mu \mathrm{g} / \mathrm{ml}$ emodin. Based on these findings, emodin concentration from 0 to $40 \mu \mathrm{g} / \mathrm{ml}$ were used to investigate the concentration-dependent effects of emodin on ECM synthesis and the activation of MAPK signals.

Emodin slightly inhibited the expression of collagen type III and fibronectin in NRK-49F cells without TGF- $\beta 1$ stimulation. The effects of emodin on the synthesis of collagen type III and fibronectin were investigated in NRK-49F cells using ELISA. Emodin slightly reduced fibronectin and collagen type III expression as compared to normal control cells (Fig. 2).

Emodin significantly inhibited ECM synthesis in TGF- $\beta 1$ stimulated NRK-49F cells. The effects of emodin on the synthesis of collagen type III and fibronectin were then examined in TGF- $\beta 1$-stimulated NRK-49F cells using ELISA. Emodin inhibited the synthesis of collagen type III in a dosedependent manner, and significantly suppressed fibronectin synthesis (Fig. 3).

Effects of MAPK inhibition on ECM synthesis. Our previous study indicated that there is no crosstalk between ERK1/2, p38 and JNK in TGF- $\beta 1$-stimulated NRK-49F cells (17). Here, we blocked each of these three MAPK pathways using their specific inhibitor to investigate the role of MAPK in ECM synthesis. PD98059 and SB202190 significantly reduced fibronectin and collagen type III synthesis in TGF- $\beta 1$-stimulated NRK-49F cells. SP600125 did not significantly suppress the secretion of fibronectin and collagen type III (Fig. 4). 

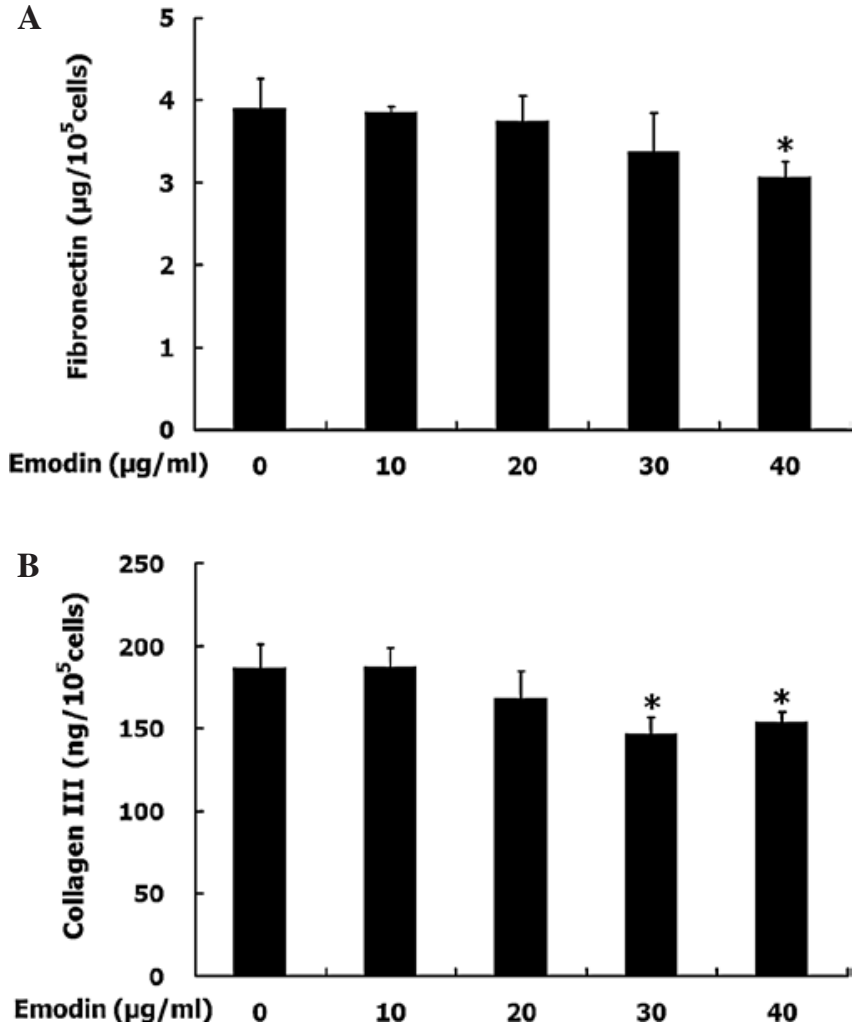

Figure 2. Fibronectin and collagen type III protein were inhibited in emodin-treated NRK-49F cells without TGF- $\beta 1$ stimulation. All results are representative of three independent experiments. Values are expressed as the means \pm SD. Fibronectin and collagen type III protein were analyzed using ELISA. Cells were incubated with emodin for 24 h. (A) Fibronectin protein in the cell culture supernatant of emodin-treated NRK-49F cells. (B) Collagen type III in the cell culture supernatant of emodin-treated NRK-49F cells. ${ }^{*} \mathrm{P}<0.05$ vs. cells without emodin treatment.

Emodin inhibited the phosphorylation of ERK1/2 and p38. Our previous study demonstrated that TGF- $\beta 1$ activates ERK1/2, p38 and JNK in NRK-49F cells (17). Here, the effects of emodin on the phosphorylation of MAPK were investigated using Western blotting. Emodin significantly suppressed the phosphorylation of ERK1/2 and p38 in a dose-dependent manner. However, emodin did not significantly reduce JNK phosphorylation (Fig. 5).

\section{Discussion}

Emodin has been demonstrated to inhibit the fibrotic process in various organs, and down-regulated TGF- $\beta 1$ and $\alpha$-SMA in an experimental rat model of hepatic fibrosis induced by carbon tetrachloride (18). Emodin also inhibits TGF- $\beta 1$ induced tissue inhibitors of metalloproteinase (TIMP-1) by suppressing activated protein-1 (AP-1) and ERK1/2 phosphorylation in hepatic stellate cells (19), and attenuates the pulmonary fibrosis process in bleomycin-treated mice and decreases fibroblast proliferation and collagen production (3). Emodin additionally suppresses TNF- $\alpha$-induced matrix metalloproteinases by blocking the AP-1 signal, as well as by inhibiting ERK and JNK phosphorylation (20).

Emodin plays a role in attenuating the fibrotic process in kidney disease. It reduces fibronectin expression by suppressing p38 signaling in diabetic rats and in high glucose-treated
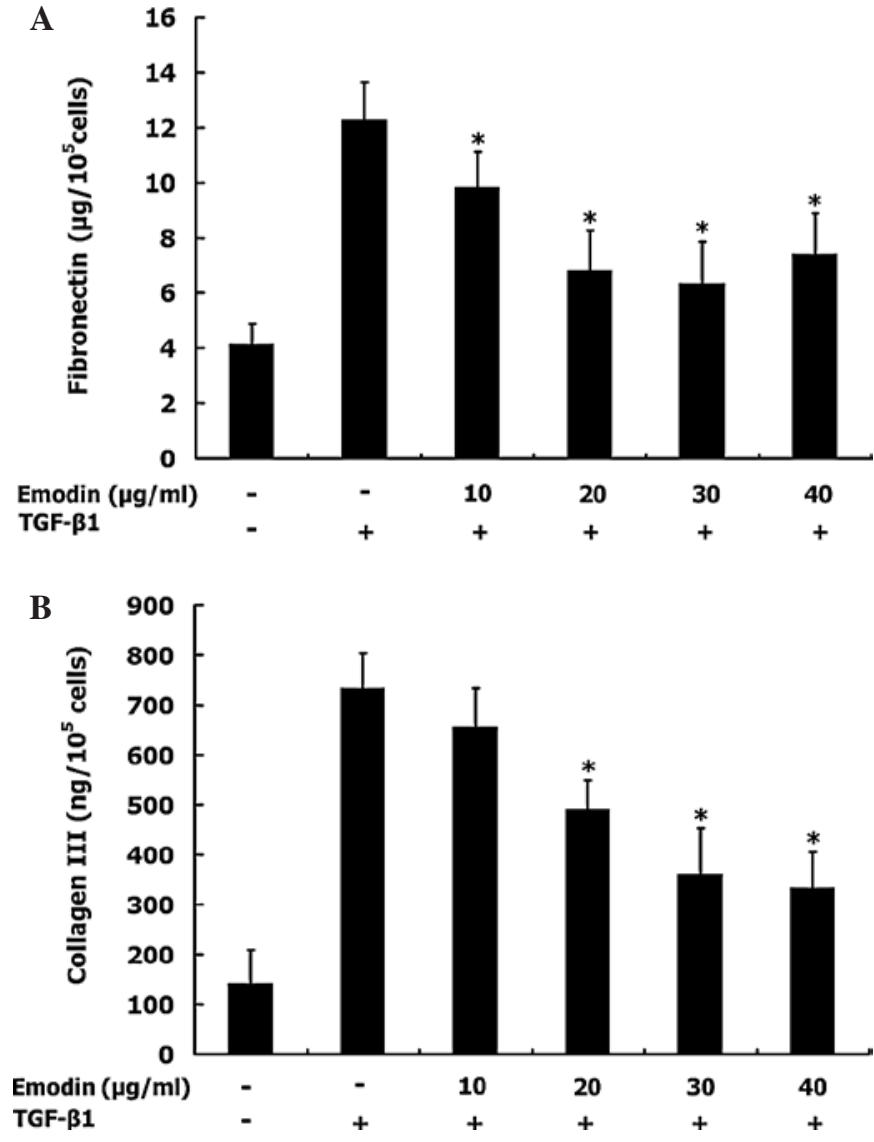

Figure 3. Fibronectin and collagen type III secretion was significantly inhibited by emodin in TGF- $\beta 1$-stimulated NRK-49F cells. All results are representative of three independent experiments with similar results. Values are expressed as the means \pm SD. Fibronectin and collagen type III protein were analyzed using ELISA, and emodin was added to the cells $2 \mathrm{~h}$ prior to $24-\mathrm{h}$ stimulation by TGF- $\beta 1$. (A) Fibronectin protein in the cell culture supernatant of TGF- $\beta 1$-stimulated NRK-49F cells treated with emodin. (B) Collagen type III in the cell culture supernatant of TGF- $\beta 1$-stimulated NRK-49F cells treated with emodin. ${ }^{*} \mathrm{P}<0.05$ vs. cells stimulated with TGF- $\beta 1$.

mesangial cells $(4,21)$. Emodin also suppresses IL-1 $\beta$-induced ECM production in mesangial cells (5) and inhibits collagen I expression in rat fibroblasts (22). However, its mechanisms are not fully elucidated.

Here, we demonstrated for the first time that emodin inhibits fibronectin and collagen type III synthesis in NRK-49F cells (a cell line of renal interstitial fibroblasts), with or without TGF- $\beta 1$ stimulation. However, emodin suppressed fibronectin and collagen type III secretion more significantly in TGF- $\beta 1$ treated cells as compared to cells without TGF- $\beta 1$, indicating that these inhibitory effects of emodin on ECM expression were partly dependent on TGF- $\beta 1$ stimulation. TGF- $\beta 1$ is the most important cytokine stimulating renal fibrosis. We have previously demonstrated that TGF- $\beta 1$ activated the ERK1/2, p38 and JNK pathways in NRK-49F cells (17). In the present study, the suppression of p38 and ERK1/2 signaling by their specific inhibitors significantly suppressed TGF- $\beta 1$-induced fibronectin and collagen III secretion, but JNK inhibition did not significantly alter ECM expression, suggesting that TGF- $\beta 1$ promotes ECM synthesis by activating the ERK1/2 and p38 pathways, but not the JNK pathway. Emodin has been demonstrated to attenuate MAPK activation in various types 

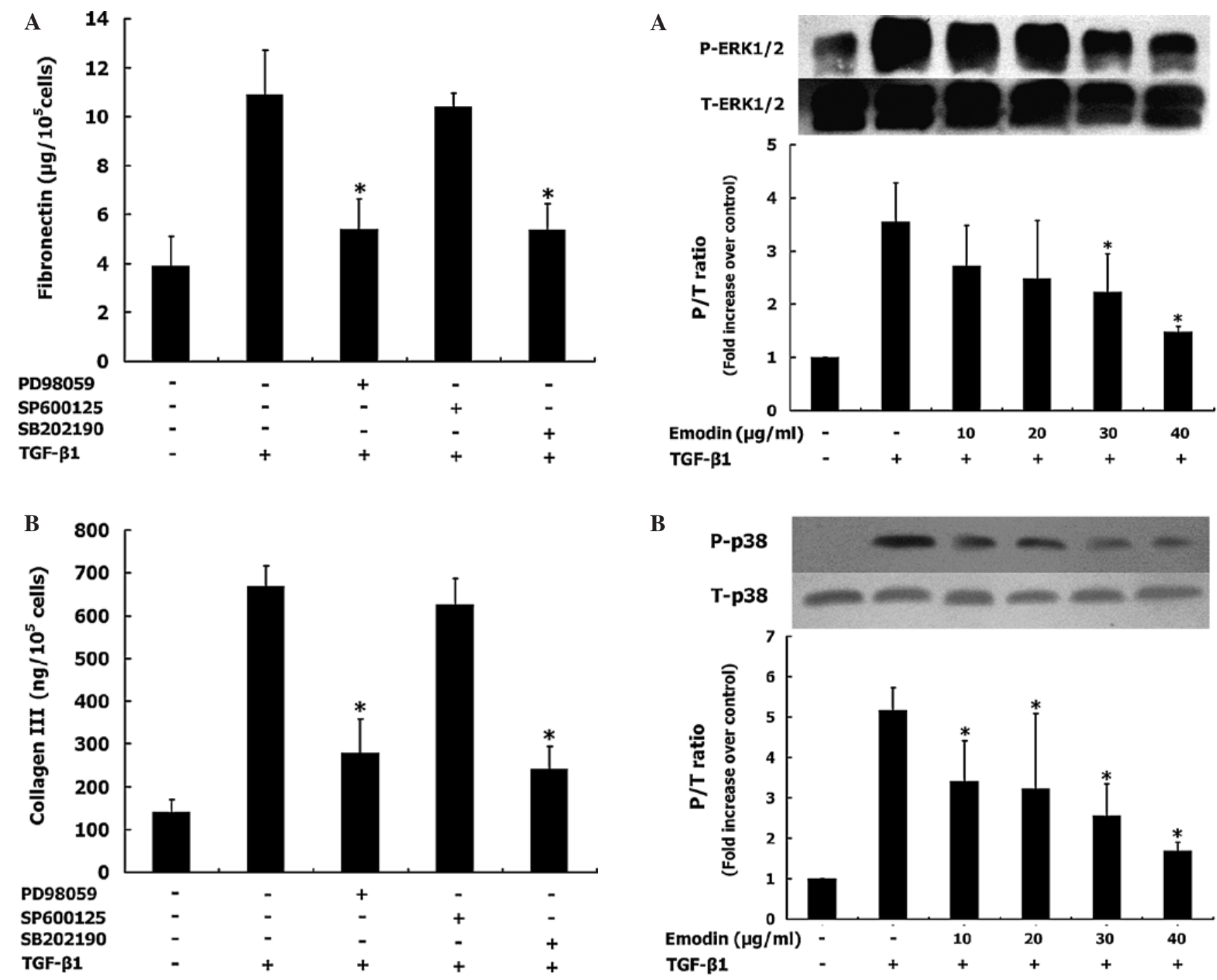

Figure 4. Fibronectin and collagen type III synthesis in TGF- $\beta 1$-stimulated NRK-49F cells treated by a specific inhibitor of p38, ERK or JNK. All results are representative of three independent experiments with similar results. Values are expressed as the means \pm SD. Fibroblasts pre-treated with PD98059 $(20 \mu \mathrm{M})$, SB203580 $(10 \mu \mathrm{M})$ or SP600125 $(20 \mu \mathrm{M})$ for $2 \mathrm{~h}$ were stimulated with TGF- $\beta 1$ for 24 h. (A) PD98059 or SB203580 significantly decreased fibronectin synthesis in TGF- $\beta 1$-stimulated cells. SP600125 did not significantly alter fibronectin synthesis. ${ }^{*} \mathrm{P}<0.05$ vs. cells stimulated with TGF- $\beta 1$. (B) PD98059 or SB203580 significantly decreased collagen type III synthesis in TGF- $\beta 1$-stimulated cells. SP600125 did not significantly alter collagen type III synthesis. "P $<0.05$ vs. TGF- $\beta 1$-stimulated cells.

of cells $(4,19,20)$. It suppresses ERK and JNK signals in HSC5 and MDA-MB-231 cells (23), and also attenuates TNF- $\alpha$ induced JNK activation in human subconjunctival fibroblasts (24). Emodin inhibits ERK1/2 to suppress hyaluronic acidinduced matrix metalloproteinase secretion (25). Emodin suppresses VEGF- $\alpha$-induced angiogenesis by blocking the phosphorylation of receptor-2, ERK1/2 and p38 (26). In SH-SY5Y cells, emodin also suppresses p38 activation (27). In IL-1-treated mesangial cells, emodin inhibits p38 and MKK3/6 phosphorylation to suppress fibronectin and collagen type IV production (5).

In this study, emodin was found to suppress TGF- $\beta 1$ induced ERK1/2 and p38 phosphorylation, but not JNK activation, suggesting that it may suppress fibronectin and

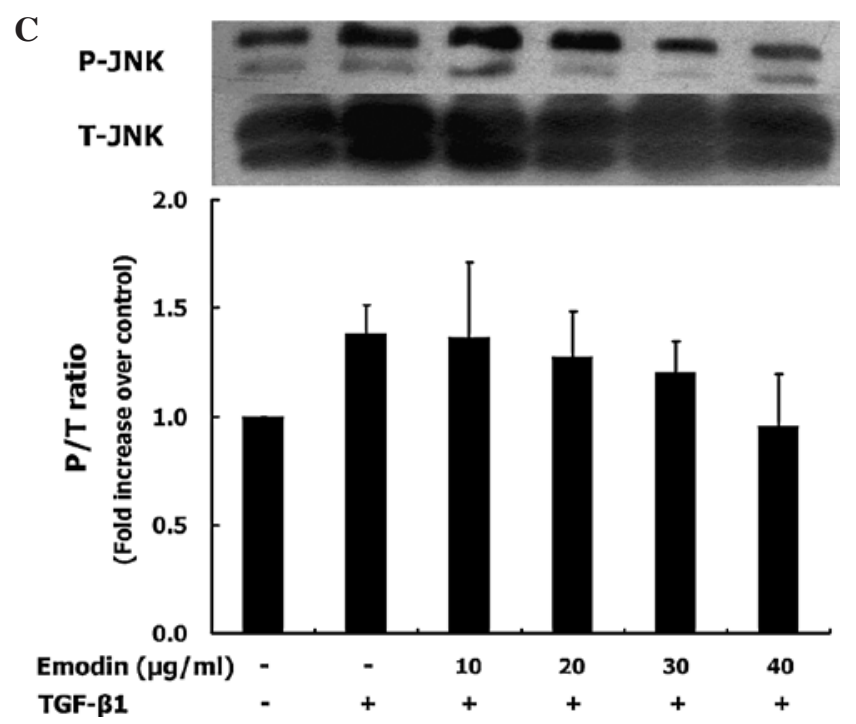

Figure 5. Effects of emodin on MAPK pathways in TGF- $\beta 1$-stimulated NRK-49F cells. The mean results of densitometric analysis of three separate experiments are shown in the bar graph. Values are expressed as the means \pm SD. Fibroblasts pre-treated with emodin for $12 \mathrm{~h}$ were stimulated with TGF- $\beta 1$ for 60 min. (A) p38 phosphorylation was inhibited by treatment with emodin. ${ }^{*} \mathrm{P}<0.05$ vs. cells stimulated with TGF- $\beta 1$. (B) ERK phosphorylation was inhibited by treatment with emodin. ${ }^{*} \mathrm{P}<0.05$ vs. cells stimulated with TGF- $\beta 1$. (C) Emodin also down-regulated JNK phosphorylation without a difference compared to cells treated with TGF- $\beta 1$ alone. 
collagen III expression by inhibiting these two signaling pathways activated by TGF- $\beta 1$. Our previous study demonstrated that there was no crosstalk between the ERK1/2 and p38 pathways (17). Therefore, emodin may suppress TGF- $\beta 1$-induced ECM expression by inhibiting ERK1/2 and P38 phosphorylation in NRK-49F cells.

In conclusion, emodin suppresses fibronectin and collagen type III expression via the inhibition of the phosphorylation of ERK1/2 and P38 in TGF- $\beta 1$-stimulated NRK-49F cells.

\section{Acknowledgements}

This study was supported by grants from the project of Hangzhou Medical Scientific Technology (no. 2005Z007), the project of the Zhejiang Natural Science Foundation (nos. Y207829 and Y2101410), the project of Zhejiang Traditional Chinese Medical Scientific Technology (no. 2005Y015) and projects in the Chinese National Science and Technology Pillar Program during the 11th 5-year plan period (no. 2006BAI04A07-1).

\section{References}

1. Zhan Y, Li D, Wei H, et al: Emodin on hepatic fibrosis in rats. Chin Med J 113: 599-601, 2000.

2. Wang $\mathrm{CH}$, Gao ZQ, Ye B, et al: Effect of emodin on pancreatic fibrosis in rats. World J Gastroenterol 13: 378-382, 2007.

3. Chen XH, Sun RS, Hu JM, et al: Inhibitory effect of emodin on bleomycin-induced pulmonary fibrosis in mice. Clin Exp Pharmacol Physiol 36: 146-153, 2009.

4. Li X, Liu W, Wang Q, et al: Emodin suppresses cell proliferation and fibronectin expression via p38MAPK pathway in rat mesangial cells cultured under high glucose. Mol Cel Endocrinol 307: 157-162, 2009.

5. Wang R, Wan Q, Zhang Y, et al: Emodin suppresses interleukin-1beta induced mesangial cells proliferation and extracellular matrix production via inhibiting P38 MAPK. Life Sci 80: 2481-2488, 2007.

6. Kutz SM, Hordines J, McKeown-Longo PJ and Higgins PJ: TGF-betal-induced PAI-1 gene expression requires MEK activity and cell-to-substrate adhesion. J Cell Sci 114: 3905-3914, 2001.

7. Secker GA, Shortt AJ, Sampson E, Schwarz QP, Schultz GS and Daniels JT: TGFbeta stimulated re-epithelialisation is regulated by CTGF and Ras/MEK/ERK signalling. Exp Cell Res 314: 131-142, 2008.

8. Nagai Y, Miyata K, Sun GP, et al: Aldosterone stimulates collagen gene expression and synthesis via activation of ERK1/2 in rat renal fibroblasts. Hypertension 46: 1039-1045, 2005.

9. Lin CL, Wang FS, Kuo YR, et al: Ras modulation of superoxide activates ERK-dependent fibronectin expression in diabetesinduced renal injuries. Kidney Int 69: 1593-1600, 2006

10. Chin BY, Mohsenin A, Li SX, Choi AM and Choi ME: Stimulation of pro-alpha(1)(I) collagen by TGF-beta(1) in mesangial cells: role of the p38 MAPK pathway. Am J Physiol 280: F495-F504, 2001.
11. Niculescu-Duvaz I, Phanish MK, Colville-Nash $\mathrm{P}$ and Dockrell ME: The TGFbeta1-induced fibronectin in human renal proximal tubular epithelial cells is p38 MAP kinase dependent and Smad independent. Nephron Exp Nephrol 105: e108-e116, 2007.

12. Sekine S, Nitta K, Uchida K, Yumura W and Nihei H: Possible involvement of mitogen-activated protein kinase in the angiotensin II-induced fibronectin synthesis in renal interstitial fibroblasts. Arch Biochem Biophys 415: 63-68, 2003.

13. Rodriguez-Pena AB, Grande MT, Eleno N, et al: Activation of Erk $1 / 2$ and Akt following unilateral ureteral obstruction. Kidney Int 74: 196-209, 2008.

14. Stambe C, Atkins RC, Tesch GH, Masaki T, Schreiner GF and Nikolic-Paterson DJ: The role of p38alpha mitogen-activated protein kinase activation in renal fibrosis. J Am Soc Nephrol 15: 370-379, 2004.

15. Ma FY, Flanc RS, Tesch GH, et al: A pathogenic role for c-Jun amino-terminal kinase signaling in renal fibrosis and tubular cell apoptosis. J Am Soc Nephrol 18: 472-484, 2007.

16. Fraser D, Brunskill N, Ito T and Phillips A: Long-term exposure of proximal tubular epithelial cells to glucose induces transforming growth factor-beta 1 synthesis via an autocrine PDGF loop. Am J Pathol 163: 2565-2574, 2003.

17. Zhu B, Wang YJ, Zhu CF, et al: Triptolide inhibits extracellular matrix protein synthesis by suppressing the Smad2 but not the MAPK pathway in TGF-\{beta\}1-stimulated NRK-49F cells. Nephrol Dial Transplant 25: 3180-3191, 2010.

18. Dong MX, Jia Y, Zhang YB, et al: Emodin protects rat liver from $\mathrm{CCl}(4)$-induced fibrogenesis via inhibition of hepatic stellate cells activation. World J Gastroenterol 15: 4753-4762, 2009.

19. Gui M, Zhang YF, Xiao ZY, et al: Inhibitory effect of emodin on tissue inhibitor of metalloproteinases-1 (TIMP-1) expression in rat hepatic stellate cells. Dig Dis Sci 52: 200-207, 2007.

20. Lee J, Jung E, Huh S, et al: Emodin inhibits TNF alpha-induced MMP-1 expression through suppression of activator protein-1 (AP-1). Life Sci 79: 2480-2485, 2006.

21. Wang J, Huang H, Liu P, et al: Inhibition of phosphorylation of p38 MAPK involved in the protection of nephropathy by emodin in diabetic rats. Eur J Pharmacol 553: 297-303, 2006.

22. Hu Q, Noor M, Wong YF, et al: In vitro anti-fibrotic activities of herbal compounds and herbs. Nephrol Dial Transplant 24: 3033-3041, 2009.

23. Huang Q, Shen HM and Ong CN: Inhibitory effect of emodin on tumor invasion through suppression of activator protein-1 and nuclear factor-kappaB. Biochem Pharmacol 68: 361-371, 2004.

24. Kitano A, Saika S, Yamanaka O, et al: Emodin suppression of ocular surface inflammatory reaction. Invest Ophthalmol Vis Sci 48: 5013-5022, 2007.

25. Kim MS, Park MJ, Kim SJ, et al: Emodin suppresses hyaluronic acid-induced MMP-9 secretion and invasion of glioma cells. Int J Oncol 27: 839-846, 2005.

26. Kwak HJ, Park MJ, Park CM, et al: Emodin inhibits vascular endothelial growth factor-A-induced angiogenesis by blocking receptor-2 (KDR/Flk-1) phosphorylation. Int $\mathrm{J}$ Cancer 118: 2711-2720, 2006

27. Lu HF, Lai KC, Hsu SC, et al: Involvement of matrix metalloproteinases on the inhibition of cells invasion and migration by emodin in human neuroblastoma SH-SY5Y cells. Neurochem Res 34: 1575-1583, 2009. 